\title{
Prognostic value of symptoms at lung cancer diagnosis: a three-year observational study
}

\author{
Dinora Polanco ${ }^{1}$, Lucía Pinilla ${ }^{1,2}$, Esther Gracia-Lavedan ${ }^{1,2}$, Anna Mas $^{1}$, Sandra Bertran ${ }^{1,2}$, Gemma Fierro $^{3}$, \\ Asunción Seminario ${ }^{1,4}$, Silvia Gómez ${ }^{1}$, Ferrán Barbé , $^{1,2}$ \\ ${ }^{1}$ Group of Translational Research in Respiratory Medicine, Hospital Universitari Arnau de Vilanova y Santa Maria, IRB Lleida, Lleida, Spain; \\ ${ }^{2}$ Centro de Investigación Biomédica en Red de Enfermedades Respiratorias (CIBERES), Madrid, Spain; ${ }^{3}$ Hospital Universitari Arnau de Vilanova, \\ Lleida, Spain; ${ }^{4}$ Hospital Joan XXIII, Tarragona, Spain \\ Contributions: (I) Conception and design: D Polanco, F Barbé; (II) Administrative support: D Polanco, G Fierro; (III) Provision of study materials \\ or patients: D Polanco, A Mas, G Fierro, A Seminario, S Gómez; (IV) Collection and assembly of data: D Polanco, A Mas; (V) Data analysis and \\ interpretation: L Pinilla, E Gracia-Lavedan, S Bertran, D Polanco, F Barbé; (VI) Manuscript writing: All authors; (VII) Final approval of manuscript: \\ All authors. \\ Correspondence to: Ferrán Barbé. Group of Translational Research in Respiratory Medicine, Biomedical Research Institute of Lleida (IRB Lleida); \\ Respiratory Department, Hospital Universitari Arnau de Vilanova and Santa Maria, Avda. Rovira Roure 80, 25198 Lleida, Spain. \\ Email: febarbe.lleida.ics@gencat.cat.
}

Background: Lung cancer is mainly diagnosed at advanced or locally advanced stages, usually when symptoms become evident. However, sometimes it may be diagnosed incidentally during routine care, while patients are still asymptomatic. Prognosis differences based on symptomatic presentation have been partially explored. Our aim was to analyze the prognostic value of the initial symptomatic state of the patients in a general lung cancer cohort.

Methods: Observational ambispective study including patients consecutively diagnosed with primary lung cancer between January 2016 and December 2018 via the lung cancer Fast Diagnostic Track (FDT). Patients were followed up until death or the end of the study in September 2019. Asymptomatic patients were compared with patients presenting symptoms. Overall survival (OS) of both groups was compared using the log-rank test. Cox regression analysis was performed to clarify the effect of the symptomatic status at diagnosis on survival. Additionally, propensity score (PS) matching analysis was performed.

Results: A total of 267 patients were analyzed; $83.5 \%$ were men, with a mean (SD) age at diagnosis of 68 (10.7) years. Incidental diagnosis was ascertained in 24.7\% of cases. Asymptomatic patients presented more frequently stage I and II disease compared to symptomatic patients (51.5\% vs. 14\%), and exhibited a significantly better prognosis, with a 3 -year OS of $63.6 \%$ (vs. 30.3\%) and a median OS that was not reached during follow-up (vs. 10.3 months). With an adjusted multivariate Cox proportional hazard model, we obtained a HR (95\% CI) of 2.63 (95\% CI, 1.6-4.2; $\mathrm{P}<0.0001)$ associated with symptomatic presentation independently of age, sex, stage at diagnosis and ECOG scale. In addition, after performing the propensity score matching analysis, the Cox regression model continued to show a significantly worse prognosis for patients presenting with symptoms $(\mathrm{P}=0.041)$.

Conclusions: Lung cancer patients who are asymptomatic at diagnosis exhibit a significantly better prognosis, regardless of the stage of the disease, underlining the importance of an early diagnosis.

Keywords: Lung cancer; prognosis; incidental diagnosis

Submitted Oct 21, 2020. Accepted for publication Jan 05, 2021.

doi: $10.21037 /$ jtd-20-3075

View this article at: http://dx.doi.org/10.21037/jtd-20-3075

(c) Journal of Thoracic Disease. All rights reserved. 


\section{Introduction}

Lung cancer is the most common cause of mortality related to cancer worldwide and represents a major health problem (1). The stage of the disease is one of the main prognostic factors and implies significant differences in terms of survival (2). Unfortunately, approximately $60 \%$ of patients are diagnosed with locally advanced or advanced stage disease, exhibiting five-year survival rates that range from $12 \%$ to $41 \%$ and are lower than $10 \%$, respectively $(2,3)$. Many factors may contribute to this late diagnosis $(4,5)$. The paucisymptomatic nature of lung cancer, especially at its initial phases, could be one of them. Early lung cancer is almost always asymptomatic, and it takes it several years to grow and produce signs or symptoms that may alert the patient $(6,7)$. When this occurs, most tumors may be at locally advanced or advanced stages, and the prognosis will be much poorer.

The potential prognostic significance of symptoms at the time of diagnosis of lung cancer has been explored in previous studies, suggesting that lung cancer patients who are asymptomatic may have a better prognosis in terms of overall survival (OS) than those who present with symptoms (8-13). However, studies specifically designed to analyze differences among these groups are limited $(8,9,13)$, and some are restricted to specific lung cancer cohorts, such as non-small cell lung cancer (NSCLC) patients (13) or surgically treated patients $(9,11,12)$. Additionally, these studies have been carried out on the basis databases from registers, surveys or hospital records, but do not specify the clinical care model from which these data have been collected.

Our work has been carried out and coordinated from the lung cancer Fast Diagnostic Track (FDT), the optimal care pathway where all patients from our region with suspicion of lung cancer are centralized to perform the diagnosis process. This consists of a structured care plan that aims to provide a management according to the up-to-date evidence with a special focus on tasks, its timing and sequence, to ensure a timely care for lung cancer patients (14). Moreover, this pathway positions us in front of a general lung cancer population from a geographic area, allowing us to perform studies with a "real-life" perspective of healthcare.

Taking this into account, our aim was to perform a population study including all patients diagnosed with lung cancer from a circumscribed area, to reflect the potential prognostic value of clinical presentation at diagnosis.

We present the following article in accordance with the
STROBE reporting checklist (available at http://dx.doi. org/10.21037/jtd-20-3075).

\section{Methods}

\section{Study design}

Observational ambispective study performed at the Lung Cancer FDT of the Respiratory Department of the University Hospital Arnau de Vilanova from Lleida, the reference tertiary hospital of our region.

\section{The lung cancer FDT}

We offer coverage to the geographic area of Lleida, which includes approximately 450,000 people and is mainly characterized by its dispersed population. Because of these demographic characteristics, our Respiratory Department launched the lung cancer FDT, a monographic consultation where all patients with suspicion of lung cancer are submitted. This consultation performs the initial diagnostic management of patients operating as an optimal care pathway. The professional team includes both a nurse manager and a pulmonologist specially dedicated to the diagnosis of lung cancer who coordinate the diagnostic process of patients. Criterion for referral is the presence of any image suspected of malignancy in both simple chest radiography or computed tomography. When the imaging modality by which patient is referred is chest X-ray, the FDT consultation requests and completes the study with a CT scan. Patients can be referred from primary care, specialized care or emergency. New cases are submitted online and reviewed by the physician in charge. If the case is considered suspected of lung cancer, it is accepted to enter the FDT, and the nurse manager provides the patient the first face-to-face appointment. If the case is not considered suspicious of neoplasia, the submission is rejected and a message is sent telematically to the doctor requesting the visit explaining the decision.

At the first visit, information about medical history, anamnesis and physical examination, is collected, and available imaging tests are reviewed. The pulmonologist draws up the diagnostic plan, requesting all subsequent diagnostic tests (radiological extension study, mediastinal staging study, techniques for pathological diagnosis and pulmonary function tests). The FDT has a particular priority in the programming of these tests and time slots specifically dedicated in the different departments. The diagnostic plan is provided in writing to the patient and 
the nurse manager schedules all the tests. At the nurse manager's office, anti-smoking health education and a brief psychological assessment to evaluate the need to refer for psychological support, are also carried out.

When all requested tests have been performed, the case is scheduled in the Thoracic Oncology MDT meeting agenda. Departments included in the MDT are Respiratory Medicine, Thoracic Surgery, Medical Oncology, Radiation Oncology, Radiology, Pathology, as well as counting with the nurse manager. The team meets on a weekly basis, assessing both cases of newly diagnosed patients for which treatment needs to be established, as well as cases of already treated patients where diagnostic or therapeutic doubts arise during follow-up. After the MDT evaluation, the FDT final visit takes place, where the results and the therapeutic strategy to be followed are reported and the patient is submitted to the subsequent treating physicians.

\section{Study population}

We included all patients assessed at the Lung Cancer FDT and diagnosed with primary lung cancer, both NSCLC and small cell lung cancer (SCLC), from January 2016 to December 2018. Exclusion criteria included patients diagnosed with thoracic malignancies other than primary lung cancer, patients without pathologic confirmation of malignancy of the suspicious lesion and patients with loss of follow-up in which survival could not be confirmed. Patients were followed up until death or until the end of the study in September 2019.

\section{Definitions}

Lung cancer was diagnosed when both a radiological image and pathological confirmation of malignancy coexisted. Incidental diagnosis was defined as a radiological finding of lung cancer on any chest image from patients without any previous lung cancer symptoms, or from chronic respiratory patients with daily respiratory symptoms, without any significant symptomatic changes that would have necessitated a medical consultation or an imaging request. If a routine chest image was performed in these patients and they reported no change in their symptoms, lung cancer was considered incidental.

\section{Development of field work and collected data}

The FDT nurse manager performed a prospective daily registry of patients evaluated at the consultation, where basic data were collected: age, sex, date of first appointment and dates of performance and results from complementary tests. Further data regarding medical history and anamnesis were collected prospectively by the physician on first visit. This physician thoroughly explored the symptomatic development of the disease, identifying the most likely first symptom of it. During the diagnostic process, data about pulmonary function, pathological results, stage of the disease and first treatment performed, were collected. Survival status was checked retrospectively by the physician using hospital and primary care electronic databases.

Clinical variables included age, sex, smoking history, comorbidities and symptom of presentation. Smoking history was categorized as absence (never smokers were defined as having smoked less than 100 cigarettes in their lifetime) (15) or presence of the habit (including both current smokers and former smokers). The quantitative assessment of tobacco exposure was expressed as pack-years. Comorbidities were identified and assessed according to the age-adjusted Charlson comorbidity index (ACCI) (16). It was calculated for each patient based on 19 diagnoses obtained from the review of the patient's medical history and adjusted for age.

The lung cancer symptom that led to initiation of the diagnostic process was defined as the symptom of presentation. Established categories were based on most frequent symptomatology referred at consultation. Symptoms could be respiratory, including cough, dyspnea, hemoptysis, chest pain and respiratory infection/exacerbation; and non-respiratory, including musculoskeletal pain, dysphagia, neurological deficit, constitutional syndrome and others less frequent. When there were no symptoms, the case was considered an incidental diagnosis, as defined above.

Pulmonary function tests (spirometry and lung volume and diffusion capacity assessments) were collected from all patients, except in patients who were not able to perform the maneuvers. All tests were performed by specialized respiratory nurses. The Masterscreen ${ }^{\mathrm{TM}}$ PFT $\left(\right.$ Jaeger $^{\mathrm{TM}}$ ), an all-in-one device capable of performing spirometry and measuring the diffusion capacity of carbon monoxide (DLCO), was used. The acceptability and reproducibility criteria followed the Spanish Society of Pulmonology and Thoracic Surgery (SEPAR) and the European Respiratory Society (ERS) guidelines $(17,18)$. A bronchodilator test was performed on all patients by administering $400 \mu \mathrm{g}$ of salbutamol in 4 puffs (100 $\mu \mathrm{g}$ per puff) in 30 -s intervals. The 
DLCO was determined by the single-breath technique (19). Collected values included the ratio of the forced expiratory volume in one second to forced vital capacity $\left(\mathrm{FEV}_{1} / \mathrm{FVC}\right)$, $\mathrm{FEV}_{1}$, and DLCO.

Lung cancer stage at the time of diagnosis was categorized according to the tumor, node, metastasis staging system corresponding to the most recent edition (8th edition) (2). Stages were defined as early-stage or localized for stage I and II; locally advanced for stage III and advanced for stage IV. Information about lung cancer histology was collected; for patients with NSCLC, the histologic subtype was recorded. The performance status of patients was assessed by the Eastern Cooperative Oncology Group (ECOG) scale. First treatment modality was also recorded, and included surgical treatment, systemic oncological treatment, radiotherapy, chemoradiotherapy and best supportive care.

At the time of data review, survival status was verified. The date of death was determined from the hospital electronic and primary care databases.

The study was conducted in accordance with the Declaration of Helsinki (as revised in 2013). The study was approved by the ethics committee of the centre (Clinical Research Ethics Committee of the Arnau de VilanovaSanta Maria Hospital University Hospital) (No. 1951) and was exempted from obtaining written informed consent from patients because of being based on anonymised data routinely collected in standard clinical practice, not affecting clinical care of patients, and the retrospective component of its design.

\section{Statistical analysis}

Descriptive statistics including the mean (SD) or median (IQR) were estimated for quantitative variables with a normal or nonnormal distribution, respectively, while absolute and relative frequencies were used for qualitative variables. Normality of the distribution was analyzed using the Shapiro-Wilks test. Participant characteristics were compared using Student's $t$-test or the nonparametric Mann-Whitney U and Kruskal-Wallis tests for skewed data. OS was defined as the time in months between the diagnosis of lung cancer and all-cause death and estimated using the Kaplan-Meier method. To compare the survival curves, the log-rank test was used. Multivariate analysis was performed using a Cox proportional hazards regression model. Additionally, propensity score (PS) matching analysis was performed using logistic regression model

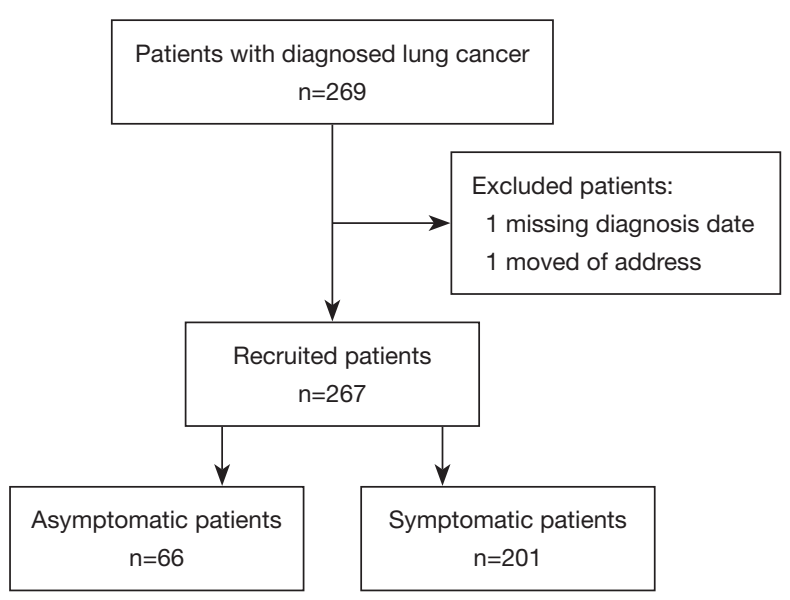

Figure 1 Flowchart of the study recruitment.

including age, sex, ECOG and disease stage as confounders. One-to-one PS matching without replacement was done to match asymptomatic to symptomatic patients and matched pairs were used in a supplemental analysis.

All tests were two-sided, and $\mathrm{P}$ values $<0.05$ were considered statistically significant. R statistical software, version 3.5.1 (R Project for Statistical Computing) was used for all analyses.

\section{Results}

We recruited 269 patients diagnosed with primary lung cancer through our Lung Cancer FDT consultation of which 267 were analyzed (Figure 1). The mean (SD) age was 68 (10.7) years at diagnosis, $86.4 \%$ of patients had a smoking history with a median [p25th;p75th] of 50 [30;68.5] pack-years. In $24.7 \%$ of patients the diagnosis of lung cancer was incidental. The OS was 11.9 months, and $61.4 \%$ of the patients died during the follow-up period.

Asymptomatic patients were significantly older, more frequently diagnosed with I and II stage disease and more frequently underwent surgical treatment as the initial approach. With respect to comorbidities, no differences were observed on the global ACCI score but, asymptomatic patients showed a higher prevalence of COPD $(\mathrm{P}=0.018)$ and cerebrovascular disease $(\mathrm{P}=0.020)$ (Table 1). Regarding clinical presentations in the symptomatic group, most patients presented respiratory symptoms (66.1\%). When it comes to stage, patients with localized stages I and II presented more frequently with exacerbation/respiratory infection, while patients with locally advanced and advanced stages III and IV showed a greater variety of symptoms 
Table 1 Clinical and demographic characteristics of the patients.

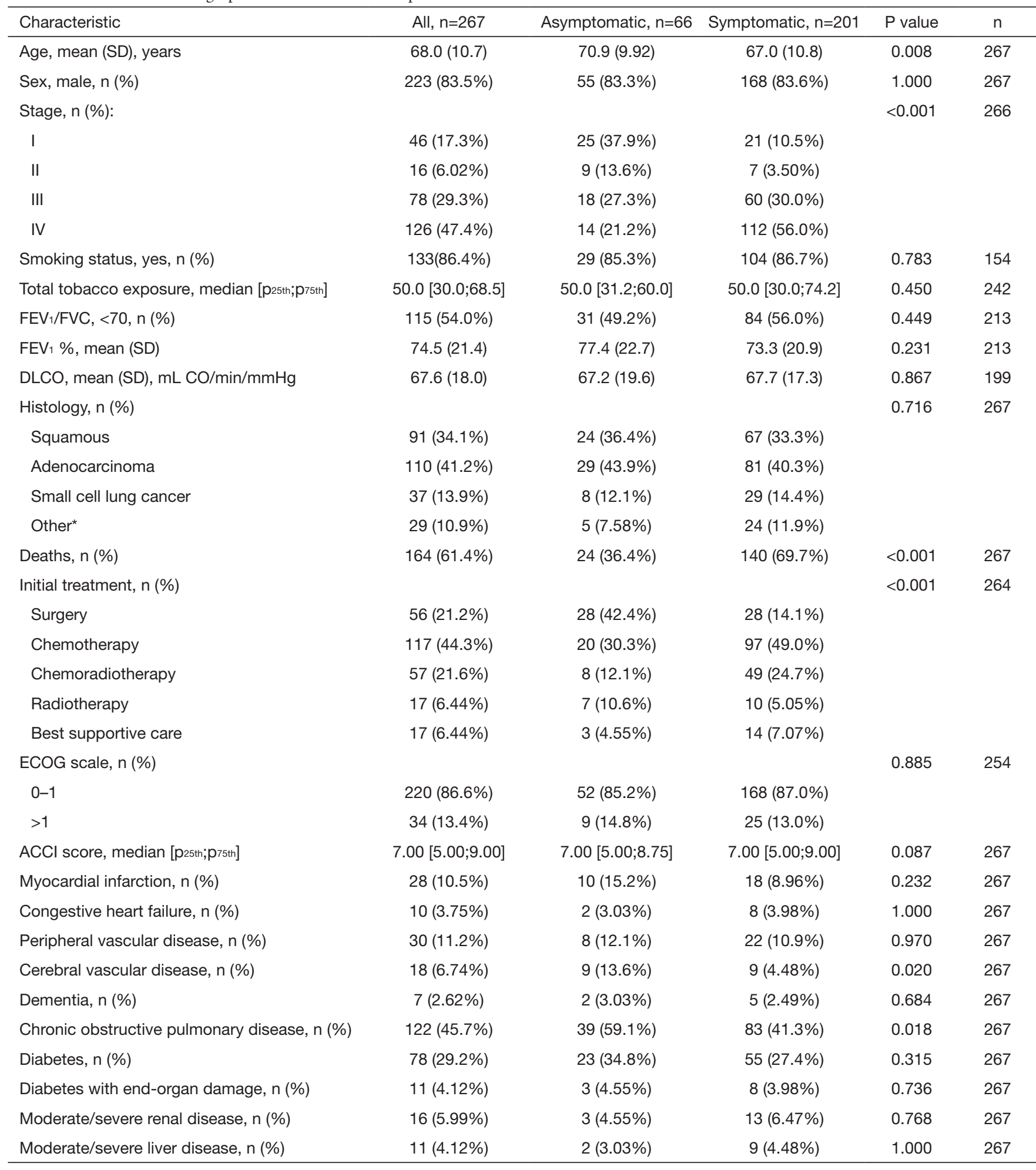

*, other histologies: large cell neuroendocrine carcinoma, carcinoid tumors (typical and atypical), adenosquamous carcinoma and undifferentiated non-small cell lung cancer-not otherwise specified (NOS). Total tobacco exposure is expressed in pack-years. SD, standard deviation; [p25th;p75th], 25 and 75 percentiles; FEV1, forced expiratory volume in 1 second; FVC, forced vital capacity. DLCO, the diffusion capacity of carbon monoxide; ECOG, Eastern Cooperative Oncology Group. ACCI, age-adjusted Charlson comorbidity index. 
Table 2 Leading symptoms in symptomatic patients categorized by stage

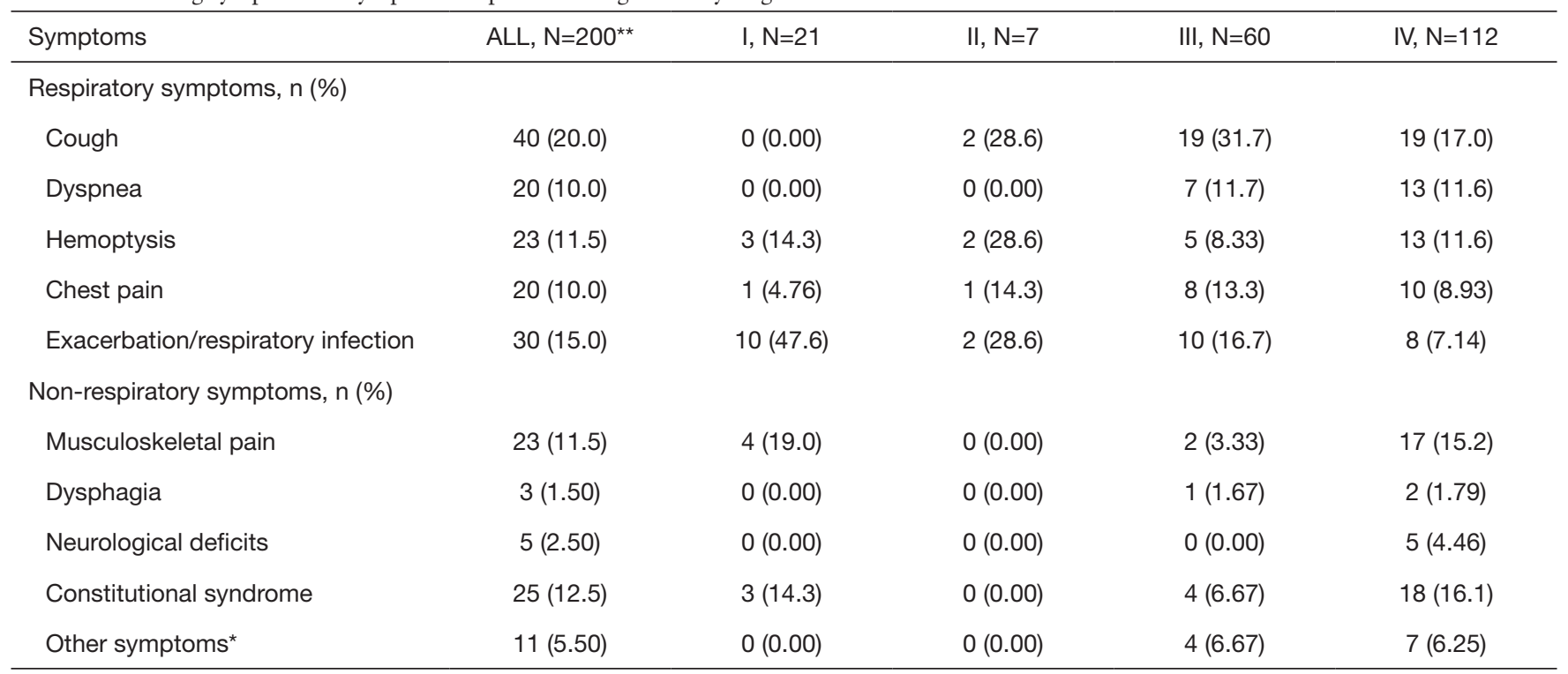

*, other symptoms: hoarseness, superior vena cava syndrome, abdominal pain and palpation of subcutaneous lesion; ${ }^{* \star}$, from symptomatic patients $(n=201)$, one has missing information about stage.

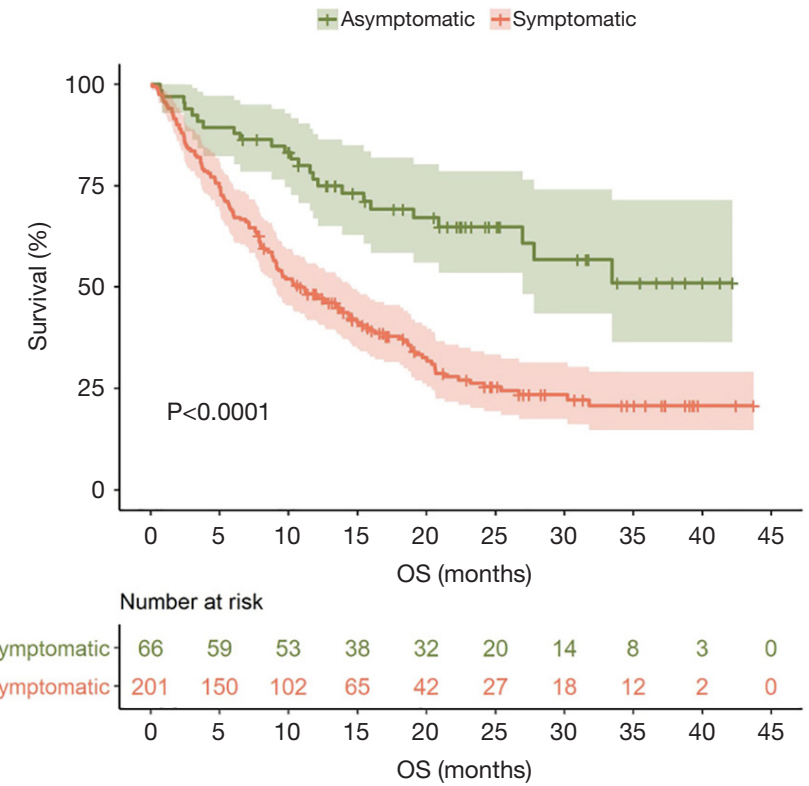

Figure 2 Kaplan-Meier curve of the time between diagnosis and all-cause death between patients who were asymptomatic and symptomatic at diagnosis. OS, overall survival.

including nonrespiratory, although the most frequent presentation was cough (Table 2).In terms of prognosis, at the end of the follow-up period, survival was significantly superior in the asymptomatic group, with a 3 -year OS of
$63.6 \%$ versus $30.3 \%$ in symptomatic patients $(\mathrm{P}<0.001)$. Accordingly, the median OS of asymptomatic patients was not achieved during the 36 months of follow-up, while in the symptomatic group, the median OS was 10.3 months $(\mathrm{P}<0.001)$ (Figure 2).

Multivariate Cox regression analysis was performed to clarify the effect of symptomatic status at the time of the initial diagnosis on survival. After adjusting the model for age, sex, disease stage and ECOG scale we obtained an HR of 2.63 (95\% CI, 1.6-4.2; $\mathrm{P}<0.0001$ ) for symptomatic versus asymptomatic patients, meaning that the risk of death was 2.63 times higher in symptomatic than in asymptomatic patients at any particular point in time (Figure 3).

As a secondary analysis, propensity score matching analysis was done to reduce the effect of potential confounding factors. No statistically significant differences were found between asymptomatic and symptomatic matched pairs (Table S1). Cox regression analysis using propensity matched patient pairs showed statistically significant worse prognosis for symptomatic patients $(\mathrm{P}=0.041)$, similar to results observed in the main analysis (Figure S1).

Differences within symptomatic patients between those presenting respiratory and non-respiratory symptoms, were also explored. Both groups presented mostly advanced and locally advanced stages at diagnosis, and no statistically significant differences were observed on survival (Table S2, 


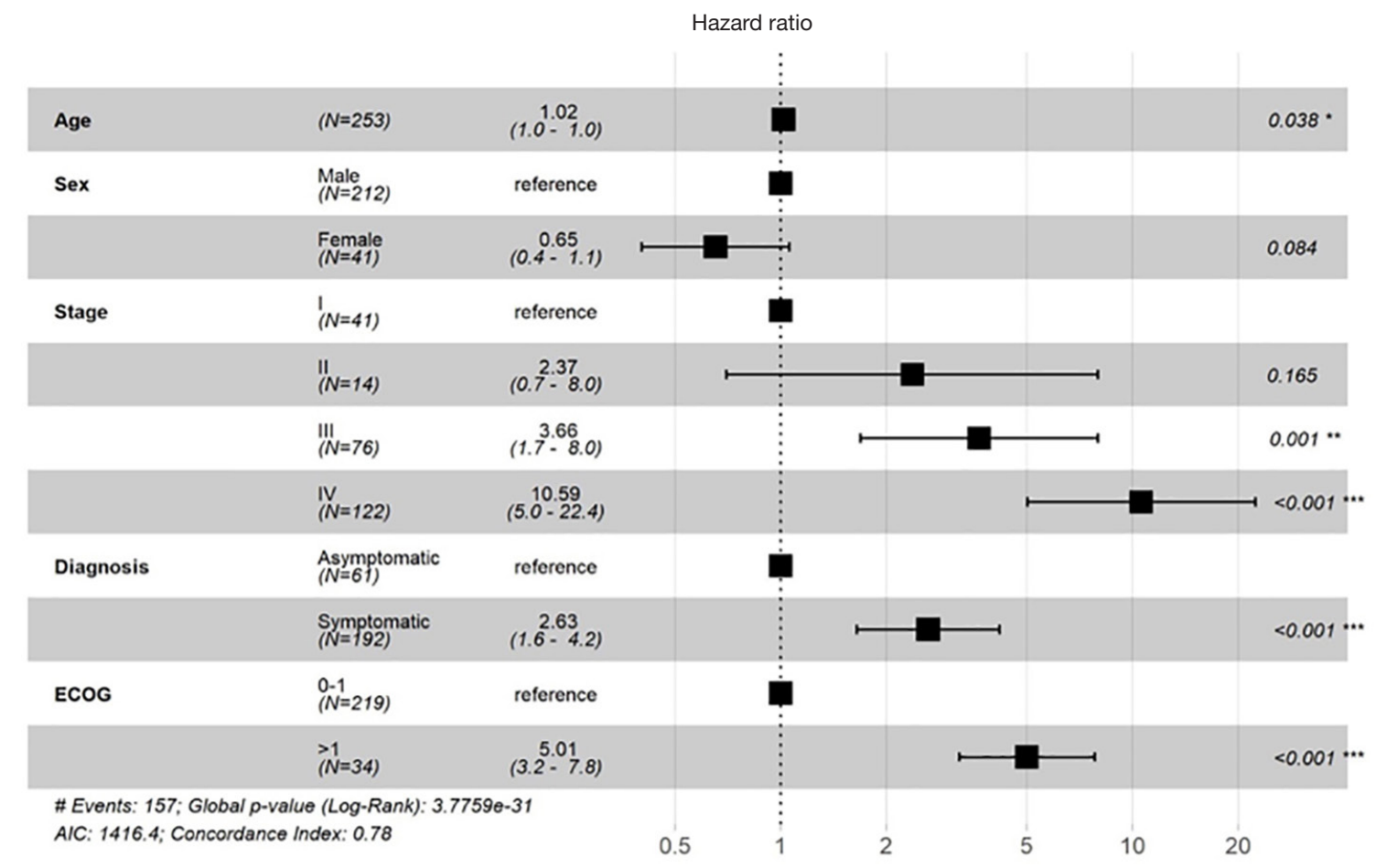

Figure 3 Hazard ratios (HR) and 95\% confidence intervals (CI) for all-cause death. Multivariate Cox regression analysis adjusted by age, sex, disease stage, symptoms at diagnosis and ECOG scale. ECOG, Eastern Cooperative Oncology Group.

Figure S2). In the multivariate Cox regression analysis both symptomatic presentations confirmed to be independent poor prognostic factors (Figure S3).

\section{Discussion}

Based on our Lung Cancer FDT registry, we observed that asymptomatic patients incidentally diagnosed with lung cancer exhibited a significantly better prognosis than those who presented symptoms at the time of diagnosis, with a 3 -year OS of $63.6 \%$ (versus $30.3 \%$ ) and a significantly superior median OS $(\mathrm{P}<0.001)$. After performing an adjusted multivariate Cox regression analysis, we confirmed that being symptomatic at the moment of diagnosis was an independent risk factor for poor prognosis, with a HR of 2.63. Additionally, propensity score matching was used to reduce selection bias, and results using matched pairs confirm our hypothesis.

In our cohort, $24.7 \%$ of patients were incidentally diagnosed. Previous studies showed differing incidences, what could be mainly due to the different lung cancer cohorts explored and different adopted definitions of incidental diagnosis. Among surgical lung cancer cohorts, there are moderate figures, ranging from $18 \%$ from Sheel et al. (11), to the $53.9 \%$ reported by Quadrelli et al. (12). In cohorts not limited to surgical patients, the prevalence of asymptomatic patients is lower, and we find values of 9.1\% from Kocher et al. (13) and 6.5\% from In et al. (10). Although performed in a general lung cancer cohort, our study shows a high prevalence of incidental diagnosis. This could be partially attributed to our definition of incidental diagnosis, particularly affecting chronic respiratory patients. Being completely asymptomatic is uncommon in these patients, therefore we considered them symptomatic only when reporting striking changes in their symptomatology. If we had considered only totally symptoms-free patients, the percentage of incidental diagnosis may have been lower and maybe similar to previously reported figures. Kocher et al. (13) preferred to use a stringent definition, and only considered patients without any potential lung cancer symptom. However, in our opinion, our criterion of "asymptomatic" sought to better reflect "real-life" daily practice with these chronic patients, which can also present their usual symptoms and hide asymptomatic incipient malignant lesions.

Also consistent with previous works, patients with 
an incidental diagnosis were significantly older $(12,13)$. Age related factors, as comorbidities that may require chest imaging and elective surgeries with their previous preoperative radiographs, may partially explain these results. This could be in line with the results observed with respect to comorbidities, as asymptomatic patients have shown a higher prevalence of COPD and cerebrovascular disease, chronic conditions that can lead to more routine imaging.

Furthermore, we observed significant differences in the initial stage at diagnosis. Incidentally diagnosed patients presented more frequently with localized stage disease, while symptomatics presented more frequently with advanced disease. This is in line with differences found in the initial treatment: asymptomatic patients more frequently underwent surgical treatment. Previous works share similar results in staging and initial treatment strategies (10-13).

We also drew the conclusion that patients incidentally diagnosed with lung cancer have a better prognosis and OS, in accordance with previous studies. Kocher et al. reported a 3 -year OS of $62.6 \%$ in asymptomatic patients compared to $30.3 \%$ in symptomatics. Additionally, they obtained an HR for a symptomatic diagnosis of 1.35 (95\% CI, 1.042-1.684; $\mathrm{P}=0.022$ ) (13). These findings, very similar to our results, clearly define a tendency for a better prognosis in these patients, so we should focus on detecting the disease prior to the development of symptoms.

In the past few years, in both the United States and Europe, screening lung cancer trials have been developed. The National Lung Screening Trial (NLST), was the first fully powered randomized controlled trial (RCT) to provide evidence of a mortality advantage in computed tomography (CT)-screened high-risk patients (20). Recently, the NEderlands Leuvens Longkanker Screenings Onderzoek (NELSON) study confirmed the findings of the NLST in a European population (21). However, for the moment, screening is not a homogeneous reality and screening procedures are widely variable in different countries. In Spain, and consequently in our hospital, there is currently no extended lung cancer screening program, so our work should be addressed to generating evidence to support this approach for early detection.

Our study has some limitations to be commented. Our follow-up time and sample were smaller than those of previous studies (8-13). This may have decreased the power of our results, and a study with a longer follow-up period and a larger sample size may have revealed greater differences. Nevertheless, our results showed significant differences and yielded similar conclusions to those drawn from prior works. Also, despite adjusting the model by disease stage and ECOG the determination of survival time among patients with incidental diagnoses may be subjected to lead-time bias. Finally, despite the fact that this model has been adjusted by variables such as stage and performance status, it has not been possible to adjust for biological parameters translating tumor aggressiveness that have been shown to be prognostic factors in patients with lung cancer (22-24).

Remarkably, our study was developed in a specific lung cancer FDT consultation, which provides high quality to clinical practice and data underlying this work. Our lung cancer FDT is an optimal care pathway. These are structured multidisciplinary care plans for specific clinical conditions, which describe the tasks to be pursued, their timing, sequence and the professionals involved (14). In brief, these pathways chart the optimal journey of patients with suspected lung cancer. Among their reported aims, one of the most significant ones is the reduction of delays experienced by patients from investigations to the initial treatment phase. This is the objective pursued by our FDT, and therefore the slogan that underlies all of our clinical practice. All of our results, diagnostic times, the patient statistics, are all reported from a consultation that aims to optimaze the medical care. In our opinion this is our strength and enhances the quality and delivery of our work. In addition, this study has been performed in a general lung cancer cohort from a circumscribed area, had an ambispective design, and was not restricted according to histological subtype or received treatment. These facts support the potential generalization and clinical implications of our results.

In conclusion, lung cancer patients who are asymptomatic at the time of diagnosis exhibit significantly better prognosis, and the presence of symptoms at diagnosis constitutes an independent risk factor for a poor prognosis. Our efforts should concentrate on continuing further research to improve and determine the key points that are lacking in the early detection of lung cancer.

\section{Acknowledgments}

We would like to thank the professionals of our multidisciplinary team for thoracic malignancies for their daily conscientious work in the diagnosis and treatment of lung cancer patients. Lucia Pinilla acknowledges receiving financial support from the Ministry of Science, Innovation 
and Universities of Spain for the Training of University Lecturers (FPU19/03526). Meetings where this work has been previously presented: (I) ERS International Congress 2019 (Madrid). Presenter: Polanco D. (II) 52nd National Congress SEPAR (Spanish Society of Pulmonology and Thoracic Surgery). Presenter: Polanco D.

Funding: None.

\section{Footnote}

Reporting Checklist: The authors have completed the STROBE reporting checklist. Available at http://dx.doi. org/10.21037/jtd-20-3075

Data Sharing Statement: Available at http://dx.doi. org/10.21037/jtd-20-3075

Peer Review File: Available at http://dx.doi.org/10.21037/jtd20-3075

Conflicts of Interest: All authors have completed the ICMJE uniform disclosure form (available at http://dx.doi. org/10.21037/jtd-20-3075). The authors have no conflicts of interest to declare.

Ethical Statement: The authors are accountable for all aspects of the work in ensuring that questions related to the accuracy or integrity of any part of the work are appropriately investigated and resolved. The study was conducted in accordance with the Declaration of Helsinki (as revised in 2013). The study was approved by the ethics committee of the centre (Clinical Research Ethics Committee of the Arnau de Vilanova-Santa Maria Hospital University Hospital) (No. 1951) and was exempted from obtaining written informed consent from patients because of being based on anonymised data routinely collected in standard clinical practice, not affecting clinical care of patients, and the retrospective component of its design.

Open Access Statement: This is an Open Access article distributed in accordance with the Creative Commons Attribution-NonCommercial-NoDerivs 4.0 International License (CC BY-NC-ND 4.0), which permits the noncommercial replication and distribution of the article with the strict proviso that no changes or edits are made and the original work is properly cited (including links to both the formal publication through the relevant DOI and the license). See: https://creativecommons.org/licenses/by-nc-nd/4.0/.

\section{References}

1. Didkowska J, Wojciechowska U, Mańczuk M, et al. Lung cancer epidemiology: contemporary and future challenges worldwide. Ann Transl Med 2016;4:150.

2. Goldstraw P, Chansky K, Crowley J, et al. The IASLC Lung Cancer Staging Project: Proposals for Revision of the TNM Stage Groupings in the Forthcoming (Eighth) Edition of the TNM Classification for Lung Cancer. J Thorac Oncol 2016;11:39-51.

3. Dingemans Martin Westeel, Virginie A-MCR. editor. Lung Cancer [Internet]. European Respiratory Society; 2015285 p. Available online: https://books.ersjournals. com/content/9781849840620/9781849840620

4. Bjerager M, Palshof T, Dahl R, et al. Delay in diagnosis of lung cancer in general practice. Br J Gen Pract 2006;56:863-8.

5. Singh H, Hirani K, Kadiyala H, et al. Characteristics and predictors of missed opportunities in lung cancer diagnosis: an electronic health record-based study. J Clin Oncol 2010;28:3307-15.

6. Geddes DM. The natural history of lung cancer: a review based on rates of tumour growth. Br J Dis Chest 1979;73:1-17.

7. Birring SS, Peake MD. Symptoms and the early diagnosis of lung cancer. Thorax 2005;60:268-9.

8. Kanashiki M, Satoh H, Ishikawa H, et al. Outcome of patients with lung cancer detected incidentally. Oncol Rep 2004;12:945-8.

9. Raz DJ, Glidden DV, Odisho AY, et al. Clinical characteristics and survival of patients with surgically resected, incidentally detected lung cancer. J Thorac Oncol 2007;2:125-30.

10. In KH, Kwon YS, Oh IJ, et al. Lung cancer patients who are asymptomatic at diagnosis show favorable prognosis: a korean Lung Cancer Registry Study. Lung Cancer 2009;64:232-7.

11. Sheel ARG, McShane J, Poullis MP. Survival of patients with or without symptoms undergoing potentially curative resections for primary lung cancer. Ann Thorac Surg 2013;95:276-84.

12. Quadrelli S, Lyons G, Colt H, et al. Clinical characteristics and prognosis of incidentally detected lung cancers. Int J Surg Oncol 2015;2015:287604.

13. Kocher F, Lunger F, Seeber A, et al. Incidental Diagnosis of Asymptomatic Non-Small-Cell Lung Cancer: A RegistryBased Analysis. Clin Lung Cancer 2016;17:62-7.e1.

14. Otty Z, Brown A, Sabesan S, et al. Optimal Care Pathways 
for People with Lung Cancer- a Scoping Review of the Literature. Int J Integr Care 2020;20:14.

15. Henschke CI, Yip R, Boffetta P, et al. CT screening for lung cancer: Importance of emphysema for never smokers and smokers. Lung Cancer 2015;88:42-7.

16. Charlson M, Szatrowski TP, Peterson J, et al. Validation of a combined comorbidity index. J Clin Epidemiol 1994;47:1245-51.

17. García-Río F, Calle M, Burgos F, et al. Spirometry. Spanish Society of Pulmonology and Thoracic Surgery (SEPAR). Arch Bronconeumol 2013;49:388-401.

18. Miller MR, Hankinson J, Brusasco V, et al. Standardisation of spirometry. Eur Respir J 2005;26:319-38.

19. Macintyre N, Crapo RO, Viegi G, et al. Standardisation of the single-breath determination of carbon monoxide uptake in the lung. Eur Respir J 2005;26:720-35.

20. Aberle DR, Adams AM, Berg CD, et al. Reduced lungcancer mortality with low-dose computed tomographic

Cite this article as: Polanco D, Pinilla L, Gracia-Lavedan E, Mas A, Bertran S, Fierro G, Seminario A, Gómez S, Barbé F. Prognostic value of symptoms at lung cancer diagnosis: a threeyear observational study. J Thorac Dis 2021;13(3):1485-1494. doi: $10.21037 /$ jtd-20-3075 screening. N Engl J Med 2011;365:395-409.

21. de Koning HJ, van der Aalst CM, de Jong PA, et al. Reduced Lung-Cancer Mortality with Volume CT Screening in a Randomized Trial. N Engl J Med 2020;382:503-13.

22. Albain KS, Crowley JJ, LeBlanc M, et al. Survival determinants in extensive-stage non-small-cell lung cancer: the Southwest Oncology Group experience. J Clin Oncol 1991;9:1618-26.

23. Hermes A, Gatzemeier U, Waschki B, et al. Lactate dehydrogenase as prognostic factor in limited and extensive disease stage small cell lung cancer - a retrospective single institution analysis. Respir Med 2010;104:1937-42.

24. Tas F, Aydiner A, Demir C, et al. Serum lactate dehydrogenase levels at presentation predict outcome of patients with limited-stage small-cell lung cancer. Am J Clin Oncol 2001;24:376-8. 
Supplementary

Table S1 Characteristics of patients of the matched paired analysis for the 1:1 propensity score

\begin{tabular}{|c|c|c|c|c|}
\hline Variable & {$[$ ALL] $\mathrm{N}=122$} & Asymptomatic $\mathrm{N}=61$ & Symptomatic N=61 & p.overall \\
\hline Sex & & & & 0.799 \\
\hline Male, n (\%) & $104(85.2 \%)$ & $51(83.6 \%)$ & $53(86.9 \%)$ & \\
\hline Female, n (\%) & $18(14.8 \%)$ & $10(16.4 \%)$ & $8(13.1 \%)$ & \\
\hline I & $40(32.8 \%)$ & $21(34.4 \%)$ & $19(31.1 \%)$ & \\
\hline II & $14(11.5 \%)$ & $8(13.1 \%)$ & $6(9.84 \%)$ & \\
\hline III & $38(31.1 \%)$ & $18(29.5 \%)$ & $20(32.8 \%)$ & \\
\hline IV & $30(24.6 \%)$ & $14(23.0 \%)$ & $16(26.2 \%)$ & \\
\hline$>1$ & $18(14.8 \%)$ & $9(14.8 \%)$ & $9(14.8 \%)$ & \\
\hline
\end{tabular}

SD: Standard deviation. ECOG: Eastern Cooperative Oncology Group.

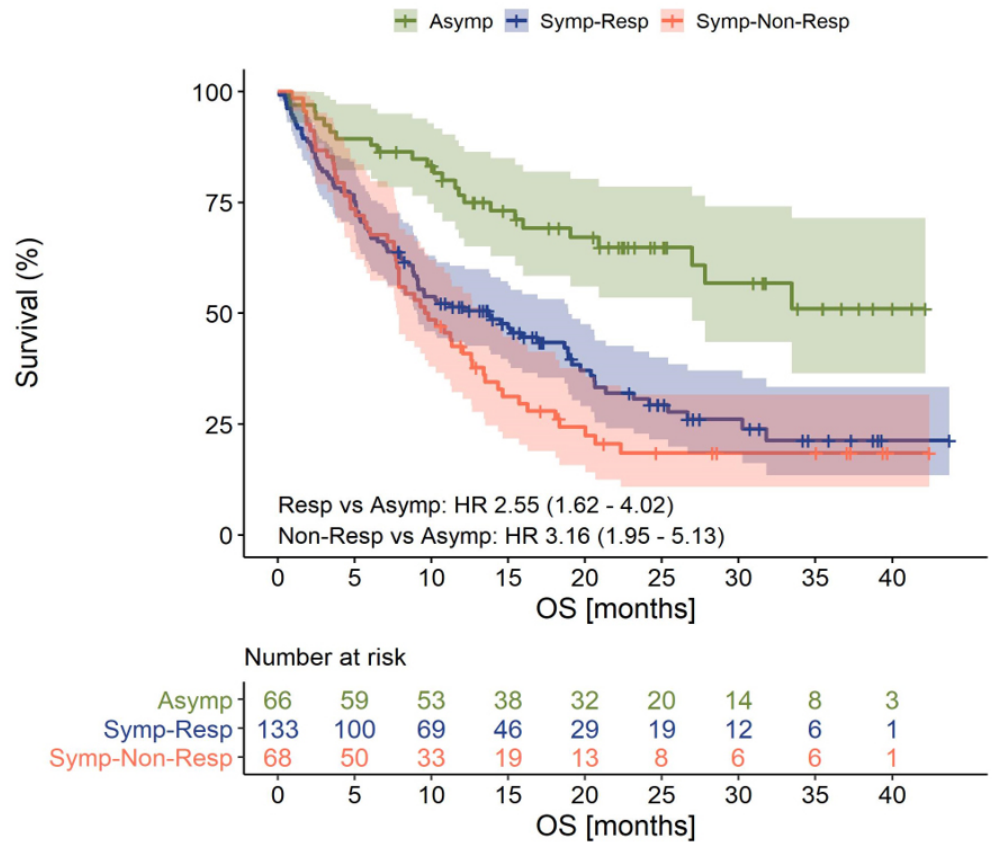

Figure S1 Kaplan-Meier curve of the time between diagnosis and all-cause death between patients who were asymptomatic and symptomatic at diagnosis, from propensity score (PS) matching analysis. HR: Hazard Ratio. OS: Overall Survival. Symp: symptomatic patients. Asymp: asymptomatic patients. 
Table S2 Clinical and demographic characteristics of patients categorized on asymptomatic, symptomatic with respiratory symptoms and symptomatic with non-respiratory symptoms

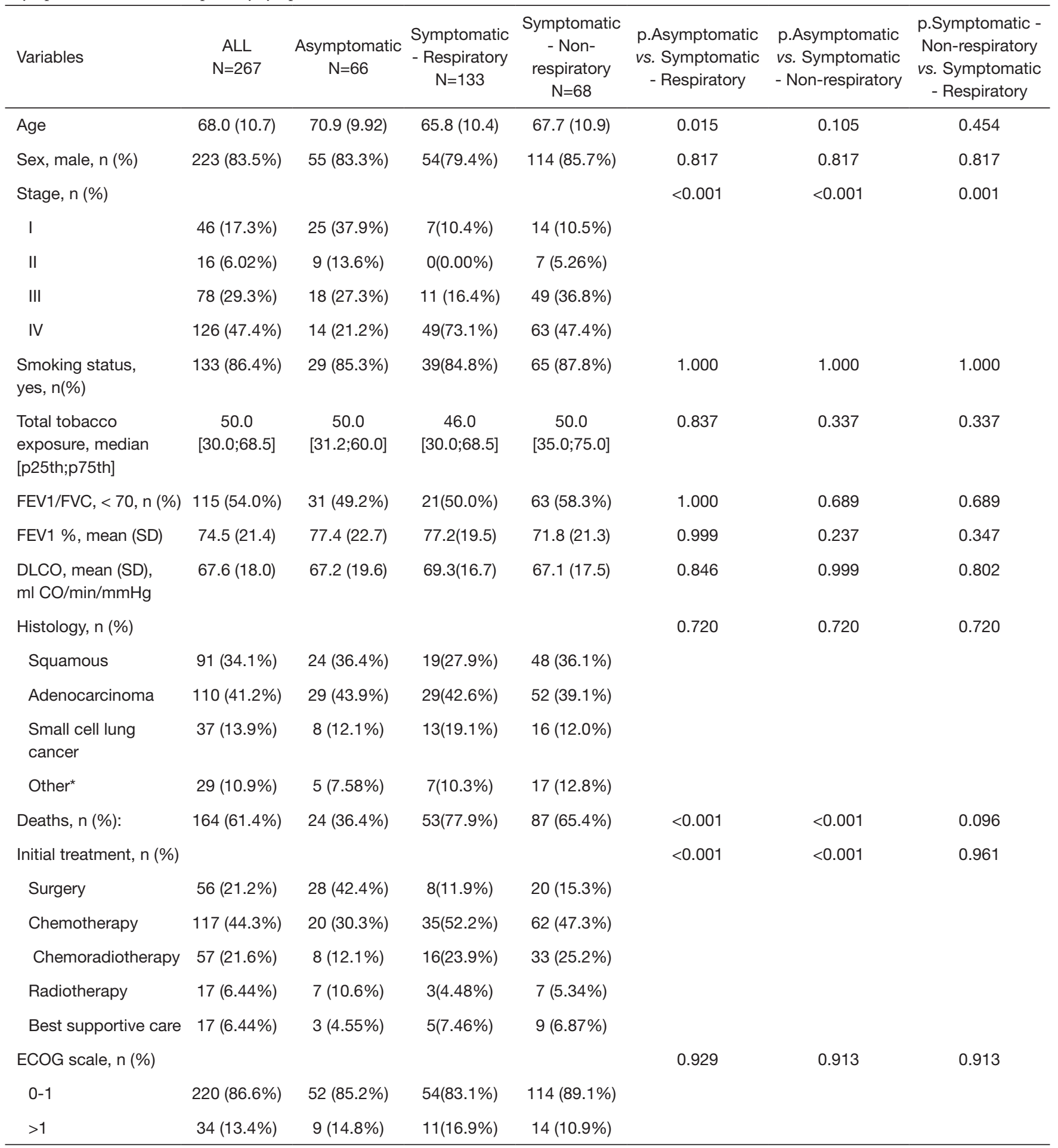

SD: Standard deviation; p25th; p75th: 25 and 75 percentiles; FEV 1 : Forced expiratory volume in 1 second; FVC: Forced vital capacity. DLCO: the diffusion capacity of carbon monoxide. ECOG: Eastern Cooperative Oncology Group. Total tobacco exposure is expressed in packyears. * Other histologies: large cell neuroendocrine carcinoma, carcinoid tumors (typical and atypical), adenosquamous carcinoma and undifferentiated non-small cell lung cancer-not otherwise specified (NOS). 


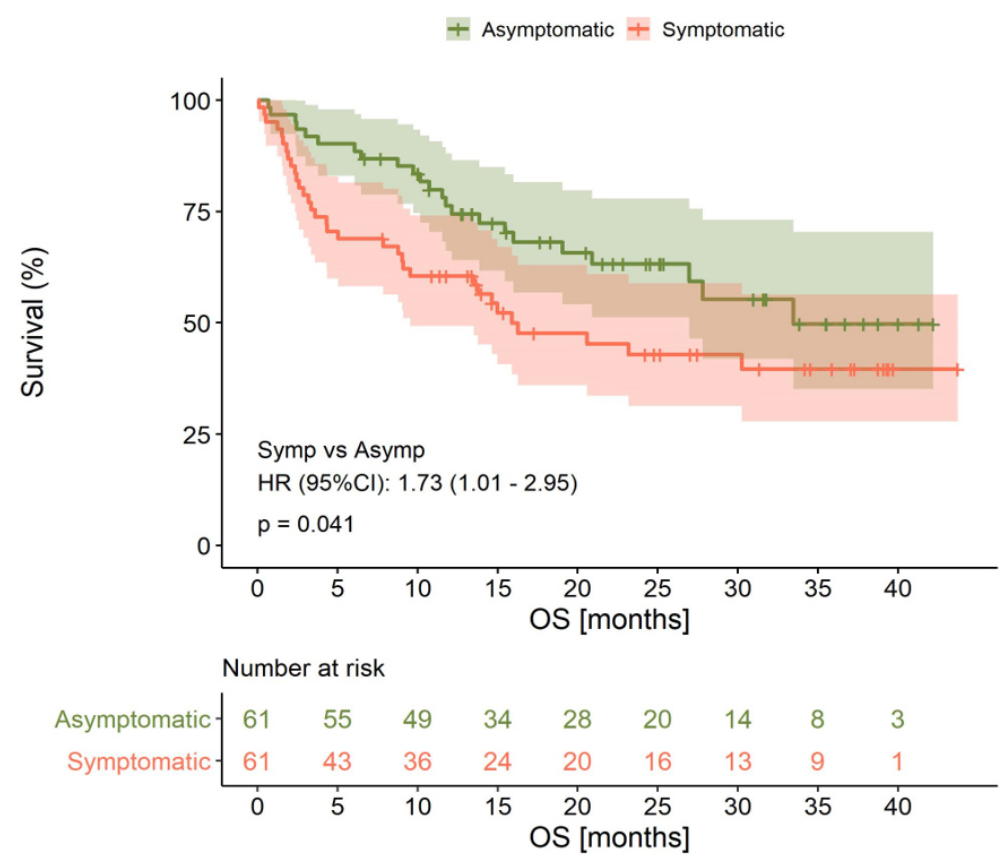

Figure S2 Kaplan-Meier curve of the time between diagnosis and all-cause death between patients who were asymptomatic, symptomatic with respiratory symptoms and symptomatic with non-respiratory symptoms at diagnosis. Resp: symptomatic patients with respiratory symptoms. Asymp: asymptomatic patients. Non- Resp/ Symp-Non-Resp: symptomatic patients with non-respiratory symptoms. HR: Hazard Ratio. OS: Overall Survival.

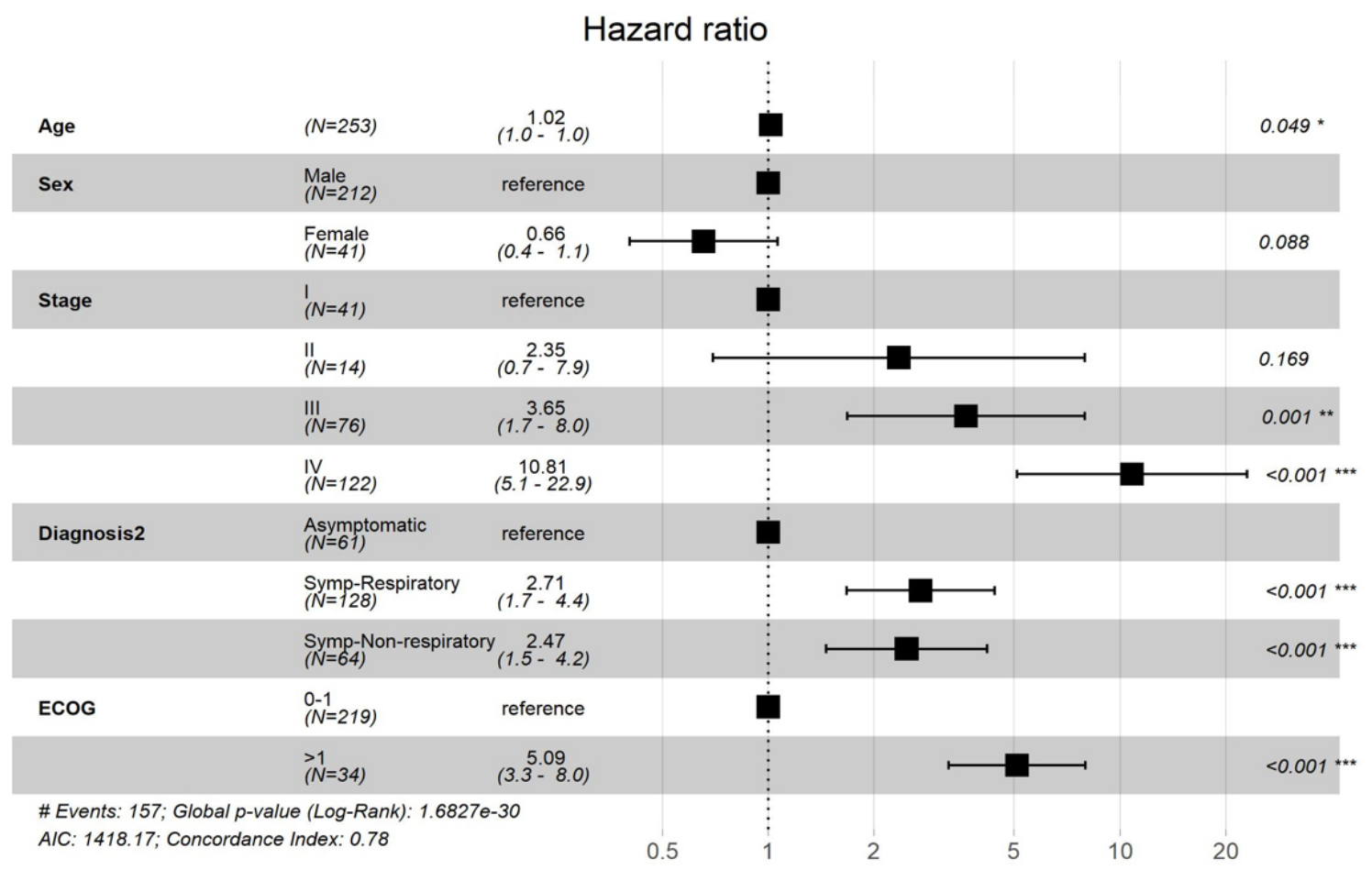

Figure S3 Multivariate Cox regression analysis adjusted by age, sex, disease stage, symptoms at diagnosis and ECOG scale. ECOG: Eastern Cooperative Oncology Group. 